\title{
A Link between Two-Sided Power and Asymmetric Laplace Distributions: with Applications to Mean and Variance Approximations
}

\section{Samuel Kotz ${ }^{1}$ and J. René van Dorp ${ }^{2}$}

The George Washington University, USA

\begin{abstract}
Motivated by our investigations of refinements of the Project Evaluation and Review Technique (PERT), we have developed a reparameterization of the asymmetric Laplace distribution and found it to be an useful tool for extending and improving various three-point approximations of continuous distributions (pioneered by Pearson and Tukey, 1965) by specifying the values of two quantiles and the mode.
\end{abstract}

KEYWORDS: Lower and Upper Quantiles, Bounded Support, Triangular Distribution, Beta Distribution

\section{INTRODUCTION}

The classical representation of the three-parameter asymmetric Laplace distribution is given by the density

$$
f(x \mid k, \sigma, m)=\frac{\sqrt{2}}{\sigma} \frac{\kappa}{1+\kappa^{2}} \begin{cases}\exp \left\{\frac{\sqrt{2}}{\kappa \sigma}|x-m|\right\} & x<m \\ \exp \left\{-\frac{\sqrt{2} \kappa}{\sigma}|x-m|\right\} & x \geq m\end{cases}
$$

with $\sigma>0$ (a scale parameter), the mode $m \in \mathbb{R}$ and $\kappa>0$ (see, e.g., Hinkley and Revankar, 1977, Kozubowski and Podgórski, 2000, and Kotz et al., 2001). An alternative parameterization is in terms of the parameters $\mu, \sigma$ and $m$ where

\footnotetext{
${ }^{1}$ Samuel Kotz, Department of Engineering Management and Systems Engineering, School of Engineering and Applied Science, The George Washington University, 1776 G Street, NW, Suite 110, Washington D.C. 20052. Email:kotz@gwu.edu.

2 J. René van Dorp, Corresponding Author, Same address, E-mail: dorpjr@gwu.edu.
} 


$$
\mu=\frac{\sigma}{\sqrt{2}}\left(\frac{1}{\kappa}-\kappa\right) \text { or } \kappa=\frac{\sqrt{2 \sigma^{2}+\mu^{2}}-\mu}{\sigma \sqrt{2}} .
$$

(See Kotz et al., 2001, for details.) Note that in both parameterizations (1) and (2) the parameters $\kappa$ and $\mu$ have no apparent interpretation.

Another recently discovered three-parameter reparameterization (see Kotz and van Dorp, 2004) involves the mode $m$ and two quantiles $a_{p}$ and $b_{r}$ such that

$$
0<p<1,0<r<1, a_{p}<m<b_{r}
$$

and the ratio

$$
\delta=\frac{m-a_{p}}{b_{r}-a_{p}}
$$

that follows from (3). Note that $\delta$ is the relative distance of the mode $m$ to the lower quantile $a_{p}$ over the range from $a_{p}$ to the upper quantile $b_{r}$ and thus $\delta \in(0,1)$. We have for the pdf of the reparameterized asymmetric Laplace distribution

$$
f_{X}\left(x \mid a_{p}, m, b_{r}\right)= \begin{cases}q(\infty) \mathcal{A} \operatorname{Exp}\{-\mathcal{A}(m-x)\} & x \leq m \\ \{1-q(\infty)\} \mathcal{B} \operatorname{Exp}\{-\mathcal{B}(x-m)\} & x>m\end{cases}
$$

where the coefficients $\mathcal{A}$ and $\mathcal{B}$ are

$$
\mathcal{A}=\frac{\log \left\{\frac{q(\infty)}{p}\right\}}{m-a_{p}} \text { and } \mathcal{B}=\frac{\log \left\{\frac{1-q(\infty)}{1-r}\right\}}{b_{r}-m}
$$

and the constant $q(\infty)$ in $(5)$ is the unique solution of the equation

$$
\frac{q(\infty)}{\delta} \log \left\{\frac{q(\infty)}{p}\right\}=\frac{1-q(\infty)}{1-\delta} \log \left\{\frac{1-q(\infty)}{1-r}\right\}
$$

From (5) it follows that $q(\infty)$ is the probability mass to the left of the mode $m$. The motivation for the designation $q(\infty)$ of this probability mass will be given in Section 3 . Reparameterization (5) is found to be useful for extending three-point approximations of arbitrary continuous distributions of the PearsonTukey (1965) type. In this note, we shall discuss the latter topic in Section 4 and present a link between 
(5) and Two-Sided Power distributions (8) in Section 3. In Section 2 we provide some background related to PERT (see, e.g., Winston, 1993) that led to our results in Sections 3 and 4.

\section{A PERT MOTIVATION FOR THE TSP DISTRIBUTION}

Consider a r.v. X with the cdf

$$
\operatorname{Pr}(X \leq x) \equiv F_{X}(x \mid a, m, b, n)=\left\{\begin{array}{cl}
\frac{(m-a)}{(b-a)}\left(\frac{x-a}{m-a}\right)^{n} & a \leq x \leq m \\
1-\frac{(b-m)}{(b-a)}\left(\frac{b-x}{b-m}\right)^{n} & m \leq x \leq b .
\end{array}\right.
$$

The four parameters $a, b, m$ and $n>0$ in (8) are the lower bound, upper bound, the mode and shape parameters, respectively. The cdf (8) is a Two-Sided Power distribution (van Dorp and Kotz, 2002) and is denoted by $\operatorname{TSP}(a, m, b, n)$. For $n=2(n=1)$ in (8) we obtain the triangular (uniform) distribution.

In the context of PERT, Malcolm et al. (1959) utilized lower and upper bound estimates $\widehat{a}, \widehat{b}$ and a most likely estimate $\widehat{m}$ to fit a four-parameter $\operatorname{Beta}(a, b, \alpha, \beta)$ distribution given by the pdf

$$
\begin{aligned}
& f_{T}(t \mid a, b, \alpha, \beta)=\frac{\Gamma(\alpha+\beta)}{\Gamma(\alpha) \Gamma(\beta)} \frac{(t-a)^{\alpha-1}(b-t)^{\beta-1}}{(b-a)^{\alpha+\beta-1}}, \\
& a \leq t \leq b, \alpha>0, \beta>0
\end{aligned}
$$

by equating $a=\widehat{a}$ and $b=\widehat{b}$, setting

$$
\left\{\begin{array}{l}
\widehat{E}[T]=\frac{\widehat{a}+4 \widehat{m}+\widehat{b}}{6} \\
\operatorname{Var}[T]=\frac{1}{36}(\widehat{b}-\widehat{a})^{2},
\end{array}\right.
$$

and using the method of moments technique to solve for the remaining beta parameters $\alpha$ and $\beta$. Application of (10) by Malcolm et al. (1959) for an indirect elicitation of $\alpha$ and $\beta$ resulted in a vigorous discussion regarding their appropriateness that has been ongoing by now for some 40 years (see, e.g., Clark (1962), Grubbs (1962), Moder and Rodgers (1968), Keefer and Verdini (1993), Kamburowski (1997), Lau et al. (1998), among others). Kamburowski (1997) emphasizes that: "Despite the criticisms and the abundance of new estimates, the PERT mean and variance (10) can be found in almost every textbook on OR/MS and P/OM, and are employed in much project management software."

Perhaps to resolve the "controversy" surrounding the use of estimators (10), D. Johnson (1997) (and possibly also others before him) have suggested that the three-parameter triangular distribution ( $n=2$ in 
the cdf (8)) be used as an alternative to the beta distribution, since the parameters $a, m$ and $b$ in (8) are in

one-to-one correspondence with the estimates $\widehat{a}, \widehat{m}$ and $\widehat{b}$. This results in an intuitive appeal of the triangular distribution for practical applications (Williams, 1992). Extending Johnson's approach, Van Dorp and Kotz (2002) and Kotz and Van Dorp (2004) suggest a more flexible four-parameter $T S P(a, m, b, n)$ distribution (8) as a proxy for the beta distribution, in particular in problems involving an assessment of risk and uncertainty (similar to PERT).

During the last few decades it has been verified that assessment of extreme values $\widehat{a}$ and $\widehat{b}$ or quantiles in their vicinity, such as the 0.01 and 0.99 percentiles (Alpert and Raiffa, 1982), is quite often beyond one's accumulated experience. Keefer and Verdini (1993) observed that the 0.10 and 0.90 quantiles have been found to be more reliable than the extreme 0.01 and 0.99 percentiles (see Selvidge, 1980) or even the "intermediate" 0.05 and 0.95 values (Davidson and Cooper, 1980). Our investigations (Kotz and Van Dorp, 2004) to solve for the lower and upper bounds $a$ and $b$ of a TSP distribution (8) given quantile estimates and a most likely estimate, led us to the results presented in Sections 3 and 4.

\section{A LINK BETWEEN TSP AND ASYMMETRIC LAPLACE DISTRIBUTIONS}

Let $X \sim T S P(a, m, b, n)$ and suppose a lower percentile $a_{p}$ (i.e. $\operatorname{Pr}\left(X \leq a_{p}\right)=p$ ), an upper percentile $b_{r}$ and the most likely value $m$ of $X$ are pre-specified in the manner that

$$
a<a_{p}<m<b_{r}<b .
$$

To uniquely solve for the lower and uppers bounds $a$ and $b$ of (8) given the shape parameter $n$ of a TSP distribution, the standardized quantity (appearing in the $\operatorname{cdf}(8)$ )

$$
q=(m-a) /(b-a)
$$

is employed. The quantity $q$ (whose value is $\leq 1$ ) represents the relative distance of the mode $m$ to the lower bound $a$ with respect to the support $[a, b]$. It follows immediately from the $\operatorname{cdf}(8)$ that

$$
\operatorname{Pr}(X \leq m)=\frac{m-a}{b-a}=q,
$$

and thus $q$ equals also the probability mass to the left of the mode $m$. From (11) and (13) we have 


$$
0<p<q<r<1 \Rightarrow 0<\frac{p}{q}<1 \text { and } 0<\frac{1-r}{1-q}<1
$$

From (8) and the definition of $a_{p}$ it follows that

$$
a_{p}=a+(m-a) \sqrt[n]{p / q}
$$

Solving the equation (15) for $a$ and recalling the condition (11), we obtain

$$
a \equiv a(q \mid n)=\frac{a_{p}-m \sqrt[n]{p / q}}{1-\sqrt[n]{p / q}}<\frac{a_{p}-a_{p} \sqrt[n]{p / q}}{1-\sqrt[n]{p / q}}=a_{p}
$$

(We use here the notation $a(q \mid n)$ instead of $a$ to emphasize that the lower bound $a$ is a function of $q$ given the shape parameter $n$, provided the $p$-the percentile $a_{p}$ and the most likely value $m$ are specified).

Analogously to (16), we have for $m<b_{r}$ (using the notation $b(q \mid n)$ in place of $b$ )

$$
b \equiv b(q \mid n)=\frac{b_{r}-m \sqrt[n]{\frac{1-r}{1-q}}}{1-\sqrt[n]{\frac{1-r}{1-q}}}>\frac{b_{r}-b_{r} \sqrt[n]{\frac{1-r}{1-q}}}{1-\sqrt[n]{\frac{1-r}{1-q}}}=b_{r} .
$$

Substituting $a(q \mid n)$ and $b(q \mid n)$ as given by (16) and (17) into (13), we arrive at the following basic equation

$$
g(q \mid n)=q
$$

where

$$
g(q \mid n) \equiv \frac{m-a(q \mid n)}{b(q \mid n)-a(q \mid n)}=\frac{\left(m-a_{p}\right)\left(1-\sqrt[n]{\frac{1-r}{1-q}}\right)}{\left(b_{r}-m\right)\left(1-\sqrt[n]{\frac{p}{q}}\right)+\left(m-a_{p}\right)\left(1-\sqrt[n]{\frac{1-r}{1-q}}\right)}
$$

Equation (18) defines a continuous implicit function $q(n)$ with domain $n>0$, where $q(n)$ is the probability mass to the left of the mode $m$ for a given value of the shape parameter $n$. Compare with (12). (Detailed straightforward calculations leading to (19) are available from the authors upon request).

Next, one can calculate the lower bound $a\{q(n) \mid n\}[(16)]$ and the upper bound $b\{q(n) \mid n\}[(17)]$ of the TSP distribution with the cdf 


$$
\begin{aligned}
& F_{X}(x \mid a\{q(n) \mid n\}, m, b\{q(n) \mid n\}, n)= \\
& \left\{\begin{array}{cc}
\frac{m-a\{q(n) \mid n\}}{b\{q(n) \mid n\}-a\{q(n) \mid n\}}\left(1-\frac{m-x}{m-a\{q(n) \mid n\}}\right)^{n} & a\{q(n) \mid n\} \leq x \leq m \\
1-\frac{b\{q(n) \mid n\}-m}{b\{q(n) \mid n\}-a\{q(n) \mid n\}}\left(1+\frac{m-x}{b\{q(n) \mid n\}-m}\right)^{n} & m \leq x \leq b\{q(n) \mid n\},
\end{array}\right.
\end{aligned}
$$

with pre-specified percentiles $a_{p}, b_{r}$ and the mode $m$ satisfying

$$
a\{q(n) \mid n\}<a_{p}<m<b_{r}<b\{q(n) \mid n\} .
$$

We shall separately consider 4 cases: A) $n \downarrow 0$, B) $n=1$, C) $n=2$, D) $n \rightarrow \infty$.

Case A: Utilizing the continuity of $q(n), a\{q(n) \mid n\}$ and $b\{q(n) \mid n\}$ as a function of $n$, it easily follows that the cdf $(20)$ converges to the Bernoulli distribution with the probability mass

$$
q(0)=\frac{m-a_{p}}{b_{r}-a_{p}}
$$

at $a_{p}$ and the probability mass $\{1-q(0)\}$ at $b_{r}$ as $n \downarrow 0$.

Case B: For $n=1$, the $\operatorname{cdf}(20)$ is simplified to a uniform distribution with parameters

$$
a=\frac{r a_{p}-p b_{r}}{r-p}, b=\frac{(1-p) b_{r}-(1-r) a_{p}}{(r-p)}
$$

and

$$
q(1)=\frac{p b_{r}+(r-p) m_{q}-r a_{p}}{b_{r}-a_{p}}
$$

Case C: As it was already mentioned the cdf (20) simplifies to a triangular distribution for $n=2$. The probability mass $q(2)$ in Case $\mathrm{C}$ has to be solved numerically from (18)-(19).

Case D: Finally as $n \rightarrow \infty$, the cdf (20) converges to the following cdf with parameters $a_{p}, m$ and $b_{r}$ :

$$
F_{X}\left(x \mid a_{p}, m, b_{r}\right)= \begin{cases}q(\infty)\left\{\frac{p}{q(\infty)}\right\}^{\frac{m-x}{m-a_{p}}} & x \leq m \\ 1-\{1-q(\infty)\}\left\{\frac{1-r}{1-q(\infty)}\right\}^{\frac{x-m}{b_{r}-m}} & x>m\end{cases}
$$

Here, $q(\infty)$ is the probability mass to the left of the mode $m$ and the unique solution in $[p, r]$ to the equation

$$
h(q)=q
$$

where 


$$
h(q) \equiv \lim _{n \rightarrow \infty} g(q \mid n)=\frac{\delta \log \left(\frac{1-r}{1-q}\right)}{(1-\delta) \log \left(\frac{p}{q}\right)+\delta \log \left(\frac{1-r}{1-q}\right)} .
$$

In (27) the function $g(q \mid n)$ and the ratio $\delta$ are given by (19) and (4), respectively. The probability mass $q(\infty)$ may be solved from (26) - (27) or equivalently from (7) utilizing a standard bisection method (see, e.g., Press, et al., 1989) with the starting interval $[p, r]$. From the cdf $(25)$ we derive its pdf given by (5), (6) and (7). This is a mode-upper-lower quantiles reparameterization of the asymmetric Laplace distribution given by (1), presented in the beginning of the introduction.

Summarizing, we have linked via the TSP family of distributions (8), the Bernoulli distribution with probability mass $q(0)[(22)]$ at $a_{p}$ and $\{1-q(0)\}$ at $b_{r}$, the uniform distribution with support $[a, b][(23)]$, the triangular distribution ( $n=2$ in (20)) and the asymmetric Laplace distribution (5).

\subsection{Moments of the reparameterized asymmetric Laplace distribution with pdf (5)}

Moments around zero of a random variable with cdf (25) and pdf (5) with the parameters $a_{p}<m<b_{r}$ are straightforwardly obtained to be

$$
E\left[X^{k}\right]=\int_{-\infty}^{\infty} x^{k} f(x) d x=q(\infty) L(k)+\{1-q(\infty)\} R(k)
$$

where

$$
\begin{aligned}
& L(k)=\int_{-\infty}^{m} \mathcal{A} x^{k} \operatorname{Exp}\{-\mathcal{A}(m-x)\} d x=\sum_{i=0}^{k} \frac{(-1)^{i} m^{k-i}}{\mathcal{A}^{i}} \frac{k !}{(k-i) !} \\
& R(k)=\int_{m}^{\infty} \mathcal{B} x^{k} \operatorname{Exp}\{-\mathcal{B}(x-m)\} d x=\sum_{i=0}^{k} \frac{m^{k-i}}{\mathcal{B}^{i}} \frac{k !}{(k-i) !} .
\end{aligned}
$$

In particular, the mean value

$$
E[X]=w_{1}\left(a_{p}-m\right)+m+w_{2}\left(b_{r}-m\right)
$$

where "the weights" are given by

$$
w_{1}=\frac{q(\infty)}{\operatorname{Ln}\left\{\frac{q(\infty)}{p}\right\}}>0, w_{2}=\frac{1-q(\infty)}{\operatorname{Ln}\left\{\frac{1-q(\infty)}{1-r}\right\}}>0 .
$$

Similarly, somewhat more tedious, direct calculations show that the variance 


$$
\operatorname{Var}[X]=u_{1}\left(w_{1}\right)^{2}\left(m-a_{p}\right)^{2}+u_{2}\left(w_{2}\right)^{2}\left(b_{r}-m\right)^{2}+2 w_{1} w_{2}\left(m-a_{p}\right)\left(b_{r}-m\right)
$$

where "the weights" $w_{i}(i=1,2)$ are as above and the additional two coefficients $u_{i}(i=1,2)$ are given by

$$
u_{1}=\frac{1+\{1-q(\infty)\}}{q(\infty)}>0, u_{2}=\frac{1+q(\infty)}{1-q(\infty)}>0
$$

Note that expression (32) for $\operatorname{Var}[X]$ involves a mixed term containing the product of deviations $\left(m-a_{p}\right)$ and $\left(b_{r}-m\right)$. In particular, for the (symmetric) case where $r=1-p$ and $m=\left(a_{p}+b_{1-p}\right) / 2$ (the mode coinciding with the median ) we have

$$
w_{1}=w_{2}=-\{2 \log (2 p)\}^{-1}
$$

and from the general expression (30) for $E[X]$ we arrive at $E[X]=m$ (independently of the value of $p$ ). Moreover, one obtains that in this case

$$
\operatorname{Var}[X]=\left\{\frac{b_{1-p}-a_{p}}{\sqrt{2} \log (2 p)}\right\}^{2}
$$

which is reminiscent of the formula for the variance of a uniform $[a, b]$ distribution in which case

$$
\operatorname{Var}[X]=\left\{\frac{b-a}{\sqrt{12}}\right\}^{2}
$$

Evidently, $\operatorname{Var}[X] \rightarrow \infty$ in $(35)$ as $p \uparrow \frac{1}{2}$ and vanishes as $p \downarrow 0$.

Similarly to the above, we set in the asymmetric case $1-r=p$ and here $m=\left(b_{1-p}-a_{p}\right) \delta+a_{p}$, $\delta \in[0,1]$. From the expression (30) for $E[X]$ we now have

$$
E[X] \equiv w_{1} a_{p}+\left(1-w_{1}-w_{2}\right) m+w_{2} b_{1-p}=a_{p}+\mathcal{C}\left(w_{1}, w_{2}, \delta\right)\left(b_{1-p}-a_{p}\right)
$$

where

$$
\mathcal{C}\left(w_{1}, w_{2}, \delta\right)=\delta\left(1-w_{1}\right)+w_{2}(1-\delta)
$$

Recalling that $r=1-p$ we rewrite $(31)$ as

$$
w_{1}=\frac{q(\infty)}{\log \{q(\infty)\}-\log (p)}>0 \text { and } w_{2}=\frac{1-q(\infty)}{\log \{1-q(\infty)\}-\log (p)}>0
$$


(Note that for $\delta=\frac{1}{2}$, we have $q(\infty)=1-q(\infty)=\frac{1}{2}$ and $E[X]$ reduces to $m$.) In a similar manner we obtain

$$
\operatorname{Var}[X]=\left\{\frac{b_{1-p}-a_{p}}{\mathcal{V}\left(w_{1}, w_{2}, u_{1}, u_{2}, \delta\right)}\right\}^{2}
$$

where

$$
\left\{\mathcal{V}\left(w_{1}, w_{2}, u_{1}, u_{2}, \delta\right)\right\}^{-2}=u_{1}\left(w_{1}\right)^{2} \delta^{2}+u_{2}\left(w_{2}\right)^{2}(1-\delta)^{2}+2 w_{1} w_{2} \delta(1-\delta)
$$

Here $u_{1}, u_{2}$ are given by (33) and $w_{1}, w_{2}$ by (39). (Compare (40) with expression (35) for the symmetric case corresponding to $\left.\delta=\frac{1}{2}\right)$. The factors $\mathcal{C}\left(w_{1}, w_{2}, \delta\right)(38)$ and $\mathcal{V}\left(w_{1}, w_{2}, u_{1}, u_{2}, \delta\right)(41)$, which can be interpreted as the mean relative distance and the variance skewness correction factors, respectively, are plotted in Figs. 1A and 1B as a function of the relative distance $\delta \in[0,1]$ of the mode to the lower quantile $a_{p}$ over the range $\left[a_{p}, b_{1-p}\right]$.
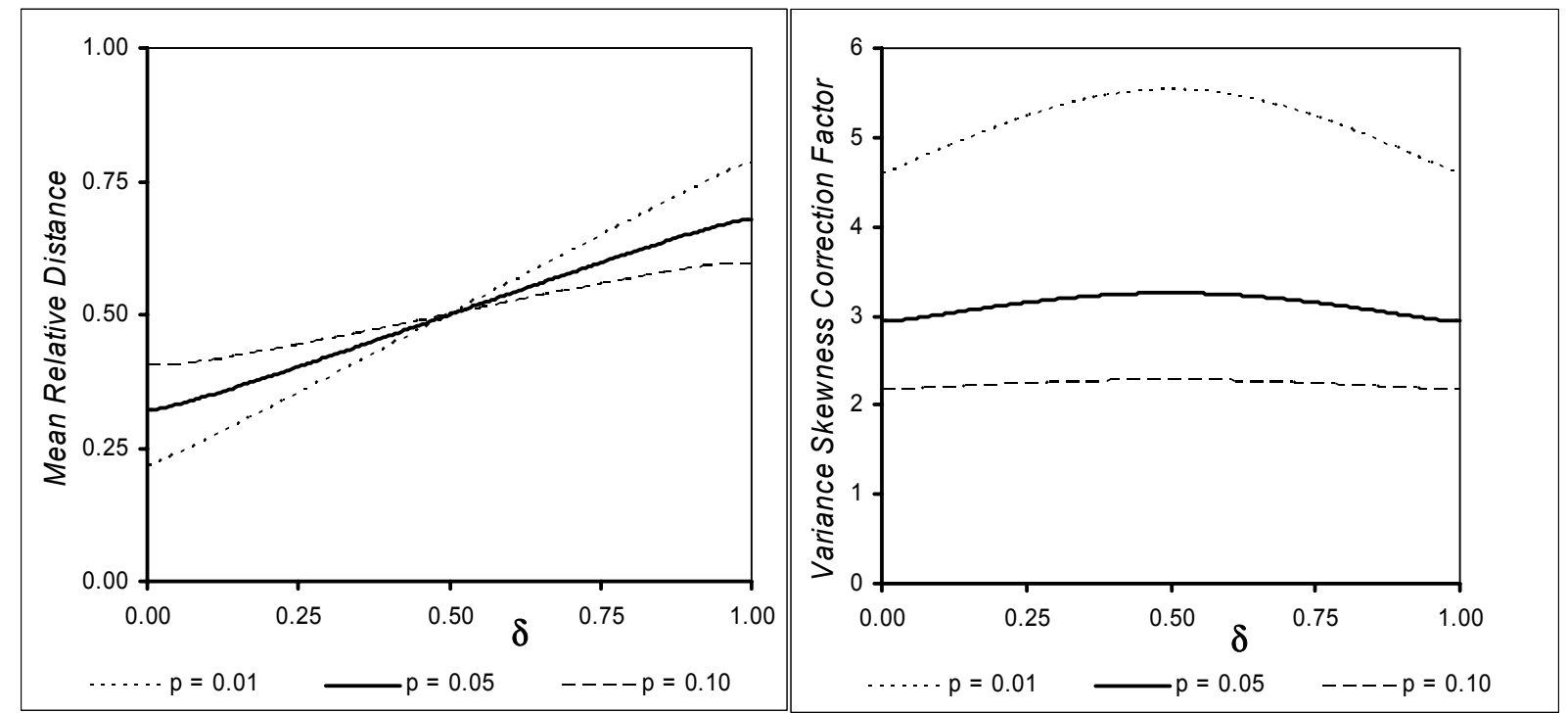

Figure 1. A: Mean relative distance as a function of $\delta(4)$

B: Variance skewness correction factor as a function of $\delta(4)$.

From Fig. 1A one observes that while the location of the mode $m$ may change from $a_{p}(\delta=0)$ to $b_{1-p}$ $\left(\delta=1\right.$ ), the mean value changes over $56 \%$ (from 0.22 to 0.78 ), $36 \%$ and $19 \%$ of the range $\left[a_{p}, b_{1-p}\right]$ for 
$p=0.01, p=0.05$ and $p=0.10$, respectively. Hence, a substantially smaller sensitivity in the mean $E[X]$ is observed due to the relative locations of the mode $m$, with respect to $a_{p}$ and $b_{1-p}$, as the tail probability $p$ increases. Recalling once again that $\delta$ is the mode relative distance it follows from Fig. 1A that for $\delta<\frac{1}{2}\left(\delta>\frac{1}{2}\right)$ the mean $E[X]$ is larger (smaller) than the mode $m$.

Figure 1B plots the variance skewness correction factor $\mathcal{V}\left(w_{1}, w_{2}, u_{1}, u_{2}, \delta\right)$. From Fig. 1B it follows that the maximum correction factor $\mathcal{V}\left(w_{1}, w_{2}, u_{1}, u_{2}, \delta\right)$ values of 5.532, 3.256 and 2.275 (and hence the minimum variance) are attained at $\delta=\frac{1}{2}$ (the symmetric case) for $p=0.01, p=0.05$ and $p=0.10$, respectively. The maximum variance (or the minimum correction factor) of an asymmetric Laplace distribution is attained at $\delta=0\left(m=a_{p}\right)$ or $\delta=1\left(m=b_{1-p}\right)$. Finally, note that similarly to the case of the mean $E[X]$, here a smaller sensitivity in the variance $\operatorname{Var}(X)$ is observed (with regard to the relative locations of the mode $m$, with respect to $a_{p}$ and $b_{1-p}$ ) when the tail probability $p$ increases.

\section{AN APPLICATION TO THREE-POINT APPROXIMATIONS}

One of the earliest and widely used three-point approximation of a continuous distributions representing a random variable $X$ in applications, with the aim to produce accurate estimates of its mean and variance, is due to Pearson and Tukey (1965). Their approximation is given as:

$$
\begin{aligned}
& E[X]= 0.185\left(a_{0.05}-x_{0.50}\right)+x_{0.50}+0.185\left(b_{0.95}-x_{0.50}\right)= \\
& 0.185 a_{0.05}+0.630 x_{0.50}+0.185 b_{0.95}, \\
& \operatorname{Var}[X]=\left\{\frac{b_{0.95}-a_{0.05}}{3.25}\right\}^{2},
\end{aligned}
$$

where $x_{0.50}$ is the median of $X$. We note that for the symmetric parameterization of the Laplace distribution where $p=1-r=0.05, m=\left(a_{p}+b_{r}\right) / 2=x_{0.50}$ we obtain from (34) that $w_{1}=w_{2}=0.217$ instead of 0.185 as given in the Pearson-Tukey approximation (42). The difference can apparently be explained by the fact that the Pearson-Tukey method was designed to approximate the mean for both symmetric and skewed distributions, not specifically the asymmetric Laplace (AL) distribution. Larger weights $w_{1}=w_{2}=0.217$ are assigned to the Laplace tails since the Laplace distribution is one of the "heavy-tails" cases (as compared to, e.g., the normal distribution). On the other hand we have for $p=0.05$ 


$$
|\sqrt{2} \log (2 p)|=3.256
$$

in (35) which agrees remarkably well with the Pearson-Tukey approximation factor of 3.25.

Keefer and Bodily (1983) suggested the three-point discrete distribution approximation with the outcomes $a_{p}, x_{0.50}$ and $b_{1-p}$ and the coefficients $0.185,0.630$ and 0.185 (interpreted as probabilities) in (42) (and a corresponding variance approximation) and termed it the Extended Pearson-Tukey method, popularized in Decision Analysis textbooks (see, e.g., Clemen and Reilly, 2001). Some other researchers have suggested using the mode $m$ rather than the median $x_{0.50}$. The general form of these approximations is

$$
\begin{aligned}
& E[X]=\pi_{1} a_{p}+\pi_{2} \mu+\pi_{3} b_{1-p} \\
& \operatorname{Var}(X)=\left(\frac{b_{1-p}-a_{p}}{\phi}\right)^{2}
\end{aligned}
$$

where $\mu$ is either $x_{0.50}$ or the mode $m,\left(\pi_{1}, \pi_{2}, \pi_{3}\right)$ sum to one and $\phi$ is a strictly positive constant. See Table 1 for several specific formulas and references and note that in Table 1 the weights $\pi_{i}, i=1,2,3$, are strictly positive. (For the three empty cells of column $\phi, \operatorname{Var}(X)$ is calculated here as the variance of an approximating three-point discrete distribution with outcomes $a_{p}, m$ and $b_{1-p}$ by interpreting the weights $\pi_{1}, \pi_{2}, \pi_{3}$ as probabilities). We shall reflect on the approximations in Table 1 using our results derived in Section 3.

Table 1. Examples of three-point approximations of the mean and the variance utilizing appearing in the literature.

\begin{tabular}{lcccccc}
\hline & $\mu$ & $\mathrm{p}$ & $\pi_{1}$ & $\pi_{2}$ & $\pi_{3}$ & $\Phi$ \\
\hline Pearson Tukey (1965) & $\mathrm{x}_{0.50}$ & 0.05 & 0.185 & 0.630 & 0.185 & 3.25 \\
Moder and Rogers (1968) & $\mathrm{m}$ & 0.05 & $1 / 6$ & $2 / 3$ & $1 / 6$ & 3.20 \\
Moder and Rogers (1968) & $\mathrm{m}$ & 0.10 & $1 / 6$ & $2 / 3$ & $1 / 6$ & 2.70 \\
Perry and Greig (1975) & $\mathrm{m}$ & 0.05 & 0.339 & 0.322 & 0.339 & \\
Megill (1977) & $\mathrm{x}_{0.50}$ & 0.10 & $2 / 7$ & $2 / 5$ & $2 / 7$ & \\
Davidson and Cooper (1980) & $\mathrm{m}$ & 0.10 & $1 / 4$ & $1 / 2$ & $1 / 4$ & 2.65 \\
Keefer and Bodily (1983) & $\mathrm{m}$ & 0.10 & 0.42 & 0.16 & 0.42 & \\
Kerzner (1992) & $\mathrm{m}$ & 0.01 & $1 / 6$ & $2 / 3$ & $1 / 6$ & 6 \\
\hline
\end{tabular}


Since the mean $E[X]$ can be viewed as the center of gravity of the probability mass, a three point approximation of it using $\left(a_{p}, \mu, b_{1-p}\right)$ should involve the distances between $a_{p}$ and $\mu, b_{1-p}$ and $\mu$, the tail probability $p$ (in both tails) and the probability masses to the left and the right of $\mu$ (as indicated in the title of Pearson and Tukey,1965). Examining the approximations of $E[X]$ as given in Table 1, we note that all values $\pi_{1}$ and $\pi_{3}$ presented therein are equal. However, those expressions approximating $E[X]$ that involve the mode $m$ can be exact only in the symmetric case, where it was verified in (34) for the symmetric Laplace case that indeed $w_{1}=w_{2}$ and hence $\pi_{1}=\pi_{3}$ (Compare (30) and (43)). For threepoint approximations of $E[X]$ involving the mode $m$ in the asymmetric case, the coefficient $\pi_{1}$ should be different from $\pi_{3}$ since the probability mass to the left of the mode $m$ does not equal that to its right.

Moreover, all the methods of variance approximations presented in Table 1 do not take into account the relative distance $\delta=\left(m-a_{p}\right) /\left(b_{1-p}-a_{p}\right)$, where as it was shown in Fig. 1B that the relative distance $\delta$ affects the value of $\operatorname{Var}(X)$ (which we believe to be the case even when $m$ is replaced by $x_{0.50}$ and $x_{0.50} \neq\left(a_{p}+b_{1-p}\right) / 2$ ). Hence, from (40) and (41) (which together form an exact expression for the variance of an asymmetric Laplace distribution in the $\left(a_{p}, m, b_{1-p}\right)$ parameterization defined in Eq. (5)), it would seem that the relative distance $\delta$ ought to enter in the construction of a three-point approximating formula for the variance of skewed continuous distributions.

Finally, in the asymmetric Laplace case and $r=1-p$ it follows from (7) that we may solve for different values of the probability mass $q(\infty)$ in (7) to the left of the mode $m$ for fixed $\delta$, for increasing values of the tail probability $p$. Figure 2 plots the modal weight $\left(1-w_{1}-w_{2}\right)$ in $(37)$ calculated via (39) as a function of the tail probability $p$ for $\delta=0.05, \delta=0.25$ and $\delta=0.5$. It follows from Figure 2 that, for example, for all values of $p \geq 0.20$ the modal weight $\left(1-w_{1}-w_{2}\right)$ is negative. This is also true for the symmetric case $(\delta=0.5)$ when the mode $m$ equals the median. Apparently, this has not been previously noticed since only values of $p \leq 0.10$ were considered in the literature (see Table 1 ). The analysis in Figure 2 is not inconsistent with the original Pearson and Tukey (1965) method, who did not interpret the coefficients in the first expression of (42) as probabilities, but calls in question this interpretation in various other approximations presented in Table 1. 
Consequently it would seem that for skewed asymmetric distributions the three-point approximations of $E[X]$ and $\operatorname{Var}(X)$ in Table 1 may not be adequate and more refined approaches are desirable involving the relative distance $\delta$ (see (37) and (38) for $E[X]$ and (40) and (41) for $\operatorname{Var}(X)$ ).

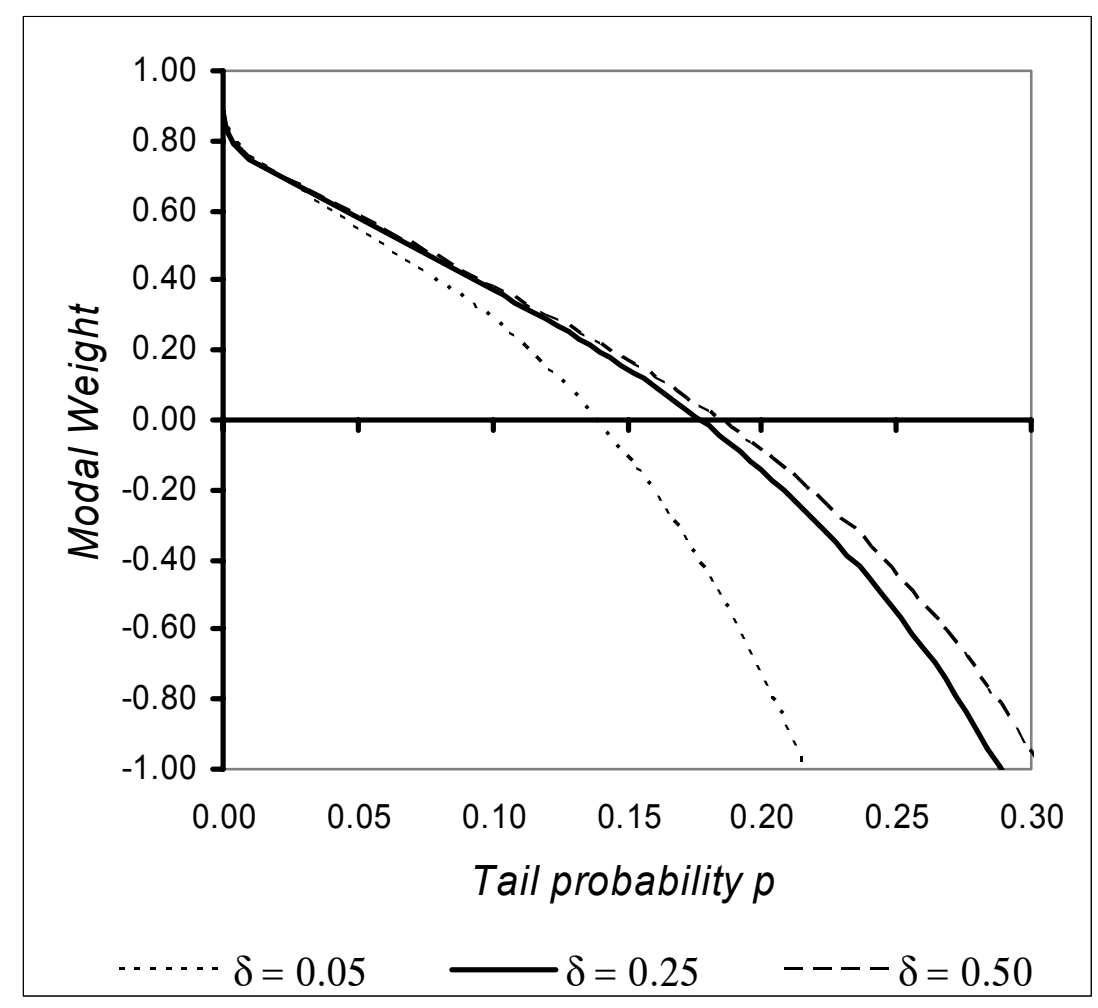

Figure 2. Modal weight $1-w_{1}-w_{2}$ in (37) as a function of the tail probability $p$ for different values of $\delta(4)$.

\section{ACKNOWLEDGEMENTS}

The authors appreciate the useful discussions with our colleagues Hernan Abeledo, Enrique CamposNanez, Thomas Mazzuchi and Professor Z. Yang of the Bejing Polytechnic University in the course of preparation of this paper. We are also thankful to the referees and the editor of Statistics and Probability Letters whose comments improved the presentation and contents of the earlier version. 


\section{REFERENCES}

Alpert, M. and H. Raiffa (1982), A progress report on the training of probability assessors, in: D. Kahneman, P. Slovic and A. Tversky, eds., Judgment Under Uncertainty: Heuristics and Biases, (Cambridge University Press, New York) pp. 294-305.

Clark, C.E. (1962), The PERT model for the distribution of an activity, Operations Research. 10, 405406.

Clemen, R.T. and T. Reilly (2001), Making Hard Decisions, with Decision Tools (Pacific Grove, Duxbury Press).

Davidson, L.B. and D.O. Cooper (1980), Implementing effective risk Analysis at Getty Oil company, Interfaces, 10, 62-75.

Grubbs, F.E. (1962), Attempts to validate certain PERT statistics or a 'Picking on PERT', Operations Research, 10, 912-915.

Hinkley, D.V. and N.S. Revankar (1977), Estimation of the Pareto law from underreported data, Journal of Econometrics, 5, 1-11.

Kamburowski, J. (1997), New validations of PERT times, Omega, International Journal of Management Science, 25 (3), 323-328.

Keefer, D.L. and S.E. Bodily (1983), Three-point approximations for continuous random variables, Management Science, 29 (5), 595-609.

Keefer, D.L. and A.V. Verdini (1993), Better estimation of PERT activity time parameters. Management Science, 39 (9), 1086-1091.

Kerzner, H. (1992), Project Management: A Systems Approach to Planning, Scheduling and Controlling (Van Nostrand Reinhold, New York, 4th ed.)

Kotz, S., T.J. Kozubowski and K. Podgórski (2001), The Laplace Distribution and Generalizations. (Boston, Birkhäuser).

Kotz, S. and Van Dorp, J.R. (2004), Input modeling of bounded uncertain phenomena for simulation and uncertainty analysis utilizing expert judgment estimates, GWU Technical Report (submitted for publication). 
Kozobowski, T.J. and K. Podgórski (2000), Asymmetric Laplace distributions, Mathematical Scientist, 25, 37-46.

Malcolm, D.G., C.E. Roseboom, C.E. Clark, and W. Fazar (1959), Application of a technique for research and development program evaluation, Operations Research, 7, 646-649.

Megill, R.E. (1977), An Introduction to Risk Analysis (Petroleum Publishing Company, Tulsa).

Moder, J.J. and E.G. Rodgers (1968), Judgment estimate of the moments of PERT type distributions, Management Science, 18 (2), B76-B83.

Pearson, E.S. and J.W. Tukey (1965), Approximate means and standard deviations based on distances between percentage points of frequency curves, Biometrika, 52 (3/4): 533-546.

Perry, C. and I.D. Greig (1975), Estimating the mean and variance of subjective distributions in PERT and decision analysis, Management Science 21, 1477-1480.

Press, W.H., B.P. Flannery, S.A. Teukolsky, W.T. Vettering (1989), Numerical Recipes in Pascal, (Cambridge University Press, Cambridge).

Selvidge, J.E. (1980), Assessing the extremes of probability distributions by the fractile method. Decision Sciences, 11, 493-502.

Van Dorp, J.R and S. Kotz. (2002), A novel extension of the triangular distribution and its parameter estimation, The Statistician, 51 (1), 63-79.

Williams, T.M. (1992), Practical use of distributions in network analysis. Journal of Operations Research Society, 43, 265-270.

Winston, W.L. (1993), Operations Research, Applications and Algorithms. (Pacific Grove, Duxbury Press, 3rd ed.). 